\title{
HIF1a blockade reduces kidney injury in lupus nephritis
}

kidney-
infiltrating
T cells from
these mice had
gene signatures
suggestive of
adaptations
to hypoxia
the kidneys in SLE by looking for clues in how the infiltrating immune cells adapt to that particular organ.

"The kidney presents unique challenges for the environmental adaptation of infiltrating immune cells as a consequence of its relative cortico-medullary and medullary hypoxia and osmolality," states Ping-Min Chen, first author of the new study. "We therefore began by asking how $\mathrm{T}$ cells that infiltrate the kidneys of mice with lupus adapt to this environment, and in turn,

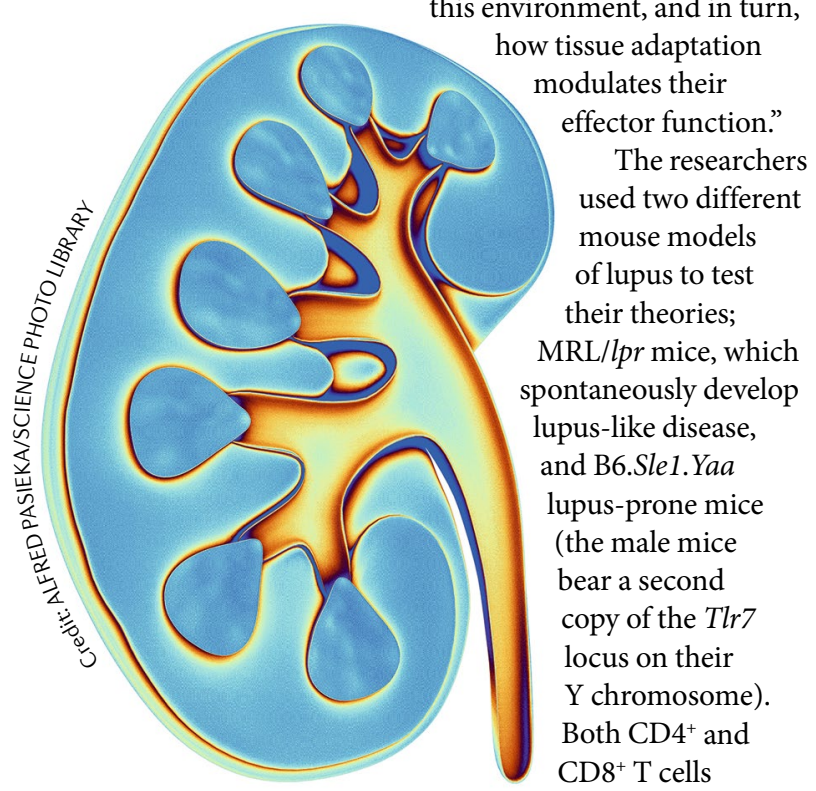

from the kidneys of these mice were transcriptionally active and pathogenic, as determined by $\mathrm{T}$ cell depletion experiments.

Kidney-infiltrating T cells from these mice had gene signatures suggestive of adaptations to hypoxia mediated by the transcription factor HIF1a. Chen and colleagues determined that this transcriptional profile was the direct result of low oxygen concentrations by injecting the mice with pimonidazole, a substance that only binds to proteins in hypoxic conditions. Double staining of kidney-infiltrating $\mathrm{T}$ cells with pimonidazole and HIF1a confirmed that these cells had adapted to the hypoxic environment, and $\mathrm{T}$ cell depletion experiments partially reversed hypoxia in the renal cortex.

"T cells are known to infiltrate the kidney, and many labs have shown that these cells have a pro-inflammatory phenotype and that manipulations (therapeutic or genetic) that lead to decreased kidney damage are linked to the decreased presence of T cells," explains George Tsokos, an expert on T cells and SLE who was not involved in this study. "The conceptual brilliance of this paper is the consideration that the extended hypoxia (which can easily be assumed given that the filtration in the glomeruli is compromised) accounts for and/or contributes to T cell-mediated kidney damage."

Using human single-cell RNA sequencing data from the Accelerating Medicines Partnership SLE project, the researchers found similar HIF1 $\alpha$-regulated gene signatures to those they found in mouse models of lupus and confirmed the expression of HIF1 $\alpha$-regulated proteins in $\mathrm{T}$ cell-rich areas of nephritic human kidneys by immunohistochemistry. Chen and colleagues then tested if HIF1a blockade (using the selective inhibitor PX-478) could ameliorate kidney damage in B6.Sle1.Yaa mice.

"We found the drug slowed the infiltration of $\mathrm{T}$ cells into kidney tissue and reversed their ability to incite tissue damage," says corresponding author Joe Craft. "These findings suggest this therapy might be beneficial for human lupus nephritis."

"Since this drug and others that block HIF1a function have been used in humans with cancer, they potentially could be available for the treatment of patients with SLE," adds Chen.

The researchers also studied lupus-prone mice that lacked HIF1a in their T cells to examine the effects of $\mathrm{T}$ cell-specific depletion of HIF $1 \alpha$ on kidney damage. The T cell-specific loss of HIFla did not affect glomerular injury and the development of proteinuria in B6.Sle1.Yaa mice, but did reduce tubulointerstitial inflammation, implicating $\mathrm{T}$ cells in only the latter stages of LN development.

"Unlike the current trend for therapeutics in SLE that suppress systemic immunity, our study suggests that targeting tissueinfiltrating immune cells is feasible as a treatment for organ damage," asserts Craft. "More detailed research on understanding how the local tissue environment modulates the effector function of tissue-infiltrating immune cells into the kidneys or other damaged organs in SLE could lead to more therapeutic options," he concludes.

Joanna Clarke

ORIGINAL ARTICLE Chen, P.-M. et al. Kidney tissue hypoxia dictates $T$ cell-mediated injury in murine lupus nephritis. Sci. Transl Med. 12, eaay1620 (2020)

RELATED ARTICLE Sharabi, A. \& Tsokos, G. C. T cell metabolism: new insights in systemic lupus erythematosus pathogenesis and therapy. Nat. Rev. Rheumatol. 16, 100-112 (2020) 